Journal of Research in Interprofessional

Practice and

Education

Vol. 6.2

2016
Journal of Research in Interprofessional Practice and Education (JRIPE)

Vol. 6.2

(C) 2016

Corresponding author: Amber Buhler. Email: abuhler@pacificu.edu

\section{Comparison of Communications Styles Among Students in Allied Health Professions Programs: How Do Our Students Communicate with Other Healthcare Providers?}

\author{
Amber V. Buhler, PhD \\ Amy E. Coplen, RDH, MS \\ Shawn Davis, PhD \\ Bobby Nijjar, EdD, RD \\ Pacific University
}

\begin{abstract}
Background: Optimal patient outcomes require communication between providers in multiple professions to initiate referrals, communicate patient treatment, and coordinate care. While there is a clear need for increased understanding of the terminology, skills, and scopes of practice of professional colleagues, these tools are of limited effectiveness if there is poor interpersonal communication between team members. Multiple bodies for Interprofessional Practice and Education (IPP/IPE) identify communication skills as an integral part of education. In fact, the third competency domain set down by the Interprofessional Education Collaborative, Interprofessional Communication, states that professionals should, "Recognize how one's own uniqueness, including experience level, expertise, culture, power, and hierarchy within the healthcare team, contributes to effective communication, conflict resolution, and positive interprofessional working relationships" [1].

Methods and Findings: As part of a required interprofessional competence course, first-year students in ten health professions programs completed the Personal Coaching Style Inventory (PSCI) to self-identify personal communication styles. A series of one-way analysis of variance (ANOVA) analyses followed by Tukey post-hoc analyses were performed in order to identify significant differences in PSCI component scores between programs. Within groups, students discussed personal and cohort-wide findings as they impact teamwork. The majority of students identified with the Mediator style. Differences in style were also found in relation to profession, gender, and race. The activity prompted discussion of varied roles in team dynamics, and how differences in style could affect interprofessional teamwork.

Conclusions: Self-awareness of personal communication styles as well as predominant styles of other health professions may enhance interprofessional communication skills. The skill with which students approach their team roles in heterogeneous groups following graduation has the potential to increase team functionality and patient outcomes.
\end{abstract}

Keywords: Communication; Interprofessional education; Team dynamics 
2

How Do Our Students Communicate with Other Healthcare Providers?

Buhler, Coplen, Davis, Nijjar

Journal of Research in Interprofessional Practice and Education

Vol. 6.2

2016

\section{Background}

Optimal patient outcomes require communication between providers in multiple professions to initiate referrals, communicate patient treatment, and coordinate care. While there is a clear need for increased understanding of the terminology, skills, and scopes of practice of professional colleagues, these tools are of limited effectiveness if there is poor interpersonal communication between team members. Multiple bodies for Interprofessional Practice and Education (IPP/IPE) identify communication skills as an integral part of education. In fact, the third competency domain set down by the Interprofessional Education Collaborative, Interprofessional Communication, states that professionals should, "Recognize how one's own uniqueness, including experience level, expertise, culture, power, and hierarchy within the healthcare team, contributes to effective communication, conflict resolution, and positive interprofessional working relationships" [1].

Communication within a healthcare team relies on several components of interaction, including well-studied institutional components [2] (technology, infrastructure, and workflow), as well as interpersonal interactions between providers. Interpersonal interactions are greatly influenced by communication styles, defined as "the characteristic way a person sends verbal, paraverbal, and nonverbal signals in social interactions denoting (a) who he or she is or wants to (appear to) be, (b) how he or she tends to relate to people with whom he or she interacts, and (c) in what way his or her messages should usually be interpreted" [3, p. 179]. The styles of communication used by physicians and nurses have been well documented and reviewed $[4,5]$, as have team dynamics/interactions within interprofessional healthcare teams $[6,7]$. However, no reports were found that examined personal communication styles as a component of broader interprofessional teams. Much of the literature on communication style comes from the communications and business management fields. It has been suggested that, like personality models, basic communication styles can be measured, and several models have been well reviewed by Aritzeta, Ayestaran, and Swailes [8] and De Vries, Bakker-Pieper, Alting Siberg, Van Gameren, and Vlug [3], most having an end-point score designating how closely the subject falls into a small number of defined categories.

Data from the communications, business management, and teaching fields show that individuals' communication styles greatly impact their ability to contribute to a high-functioning team and, therefore, to improve business and educational outcomes $[9,10,11]$. This has been appreciated within the field of nursing, which has established a rich literature and resources for interpersonal communications education [4,5], followed by a growth of communication skills units in IPE [12,13]. Considering this increased appreciation of the need for communication training, the paucity of studies of communication styles within the other allied health professions is surprising.

In this study, students in ten health professions programs self-identified their communication styles using a communication inventory tool. This activity was part of an interprofessional communication learning unit within a multi-program required course on interprofessional competency and had both educational and 
3

How Do Our Students Communicate with Other Healthcare Providers?

Buhler, Coplen, Davis, Nijjar
Journal of Research in Interprofessional Practice and Education

Vol. 6.2

2016 research goals. The educational purpose of the activity was for students to self-reflect on their own communication styles, and to explore how this could impact their interactions within learning teams and in future clinical teams with other healthcare professionals. Learning objectives for the students were to: 1) identify patterns within their own style of communication, 2) describe the impact their style has on communications with others, 3 ) identify differences in communication styles endorsed by other health professions, and 4) discuss the potential problems and benefits of heterogeneous communication styles within an interprofessional team. The research purpose of this study was to determine whether differences in communication styles exist between students in different healthcare professions.

As healthcare professionals begin to work on more interprofessional teams, a professional's understanding of how the impact of his or her own style of giving and receiving feedback can affect the success of the team is crucial. Similarly, mindfulness of communication styles predominant to particular professions has the potential to reduce interpersonal tensions, increase healthcare team effectiveness, and benefit patient outcomes.

\section{Methods}

In 2009, as part of its goal to foster interprofessional practice, the College of Health Professions (CHP) introduced the course Interprofessional Competency: Theory and Practice (IPC). This course is a requirement for all first-year students in the CHP Health Professions Programs, with two class sessions in the fall and three in the spring semester, as well as experiential hours and a poster session on an interprofessional case or topic. Each class (unit) is two hours in length, and the first four sessions each focus on one of the four interprofessional competencies set forth by the interprofessional collaborative [1]: roles and responsibilities, team dynamics and professionalism, effective communication, and appreciation of differences. The fifth class session focuses on community resources and course evaluations. Common features of the units include active learning strategies and small-group work in student teams of seven or eight individuals from different professional programs. A mixed-profession team of faculty moderates all class sessions.

At the beginning of this study, in spring 2009, the course included eight schools and programs: Audiology, Dental Hygiene, Healthcare Administration, Occupational Therapy, Physician Assistant Studies, Pharmacy, Physical Therapy, and Professional Psychology. Speech-Language Pathology and Athletic Training were added in 2013 and 2014, respectively. These ten schools and programs will be referred to here as the Health Professions Programs.

This article is based on a classroom activity completed during the "effective communication" unit incorporating three full cohorts of first-year Health Professions Program students [academic year 2012-2015]. This activity was designed to assist students in developing skills for interprofessional communication utilizing the Personal Coaching Style Inventory (PSCI). The tool was originally developed by CoachWorks International [14] (Dallas, Texas, US, for Corporate Coach U international, copyright 1995, version 1999) in the early 1990s to be utilized in coach train- 
4

How Do Our Students Communicate with Other Healthcare Providers?

Buhler, Coplen, Davis, Nijjar
Journal of Research in Interprofessional

Practice and

Education

Vol. 6.2

2016 ing courses for business managers and consultants working in organizations. The designers of the tool reviewed several long validated inventories and consulted with experts in the field of business to develop a tool to serve their specific goals. The inventory was then field-tested for a year to assess for reliability and validity. While research has not been conducted to validate this inventory in an educational setting, it has been utilized with over 10,000 individuals in the business sector and been translated into multiple languages (through personal communication with developers). The authors believe that the attention to content validity and rigorous field-testing by the measure developers, along with the continued use by professionals within the field, justifies the use of the PSCI for our purposes. Permission was received to administer the inventory within our course. The PSCI is a self-report inventory wherein the subject indicates identification with 80 traits across four distinct dimensions (Director, Presenter, Mediator, and Strategist) [14]. A brief description of each of the four dimensions is given in Table 1. In that the PSCI is a multidimensional assessment, scores for each style are viewed as separate independent dimensions operating within an individual, as opposed to being an indicator of a single preferred style. One reason the PSCI was chosen over other inventories used to categorize team role and conflict-management styles [8,9], is that while most have a cost associated, permission was granted to apply this inventory at no charge. In addition, it is a short and easily used self-inventory, providing a description of potential strengths and liabilities for each style, as well as strategies for how to tailor behaviour in order to improve efficacy of communication within a team.

\section{Table 1. Personal coaching styles inventory dimension descriptions}

\begin{tabular}{|c|l|}
\hline $\begin{array}{c}\text { Personal coaching } \\
\text { styles inventory } \\
\text { dimensions }\end{array}$ & \begin{tabular}{c} 
Description \\
\hline Director
\end{tabular} \\
\hline $\begin{array}{l}\text { Directors like to be in control. They are results oriented and possibility thinkers. They } \\
\text { eagerly take on new, often risky, challenges and are quick to set goals and work hard to } \\
\text { achieve them. They are often selected as the leader, and rail against rules laid down by } \\
\text { others, preferring instead to blaze their own trail. From their vantage point, directors can } \\
\text { see strategic advantages and orchestrate the actions to beat the competition. They tend to } \\
\text { work long hard hours. People say directors have a lot of drive, make good leaders, and are } \\
\text { assertive and outspoken. }\end{array}$ \\
\hline Mediator & $\begin{array}{l}\text { Mediators are those personable people everyone seems to like. They become experts in } \\
\text { their field and are sought out for advice because of that expertise. They are extremely } \\
\text { helpful and caring sometimes to a fault. Mediators make everyone in the group feel } \\
\text { comfortable. And, while they like people, they are generally reserved in the way they } \\
\text { communicate with others. They never like showboating so they are often thought of as } \\
\text { uninspiring by the presenters. Mediator leaders instill trust in those who work with them. } \\
\text { Patient, thorough, and tolerant of other people, mediator leaders and mediator } \\
\text { employees can move mountains, one piece at a time. }\end{array}$ \\
\hline
\end{tabular}


Table 1. (continued)

How Do Our Students Communicate with Other Healthcare Providers?

Buhler, Coplen, Davis, Nijjar
Journal of Research in Interprofessional Practice and Education

Vol. 6.2

2016

\begin{tabular}{|c|c|}
\hline $\begin{array}{l}\text { Personal coaching } \\
\text { styles inventory } \\
\text { dimensions }\end{array}$ & Description \\
\hline Strategist & $\begin{array}{l}\text { Strategists are the thorough, painstaking, hardworking tacticians. They are expert analysts } \\
\text { and problem solvers. They follow the rules and think things through slowly and carefully, } \\
\text { questioning and evaluating nearly everything and everyone. Strategists hate to be wrong } \\
\text { and they hate making mistakes. And, you always know where they stand for they are clear } \\
\text { and diplomatic communicators. Although tending to be perfectionists, you can trust that } \\
\text { no stone has been left unturned. They are tuned in to the operational/task oriented follow } \\
\text { through that is critical to each project. They will not give up until the job is complete. }\end{array}$ \\
\hline Presenters & $\begin{array}{l}\text { Presenters know everyone is important. They love to talk to anyone about anything, } \\
\text { anytime. Being animated, energetic, and spontaneous, they like to have everything they } \\
\text { do be fun. They are excellent communicators, and can be very persuasive. They often } \\
\text { embellish their experiences with colorful anecdotes that are crowd pleasers. Presenters } \\
\text { look successful to others. They like variety, are curious, and sometimes impulsive. They do } \\
\text { not like to be tied down to one thing and quickly lose interest in projects after startup. } \\
\text { People are drawn to them because of their intuitive connection. Individuals confide in } \\
\text { them easily. They are comfortable being the center of attention. }\end{array}$ \\
\hline
\end{tabular}

In preparation for classroom discussion, students were required to complete the PSCI prior to the class. The inventory was available online in the form of a Moodle questionnaire and took approximately eight minutes to complete [15]. After completion of the required portion (PSCI questions and identification of their field of study), students were directed to a second, optional portion with demographic questions pertaining to age, gender, and race. These categories align with those outlined by the National Institutes of Health (NIH) in its Policy on Reporting Race and Ethnicity Data: Subjects in Clinical Research [16]. All data was de-identified before analysis and the Pacific University Institutional Review Board granted exemption for this study (IRB exemption \#017-13).

Students received their scores for each domain immediately after completion. At this time they were also given electronic access to the accompanying materials describing the four domains and suggested communication strategies. During inseat instructional time students were first shown graphical representations (pie charts) of the distribution of communication-style dimensions in each of the represented health professions. Students were then "broken out" into pre-assigned mixedprofession learning groups and prompted to discuss: 1) Which style were each of you? Do you feel that was accurate? 2) What communication obstacles could come up for your group given the varying styles of your group members? 3) What implications do the graphs have on interprofessional communication as we begin to work on more interprofessional teams in healthcare? The additional demographic information was not shared with students during the presentation.

The hypothesis tested within the research portion of this study is that there are differences in self-identified communication styles based on healthcare profession. 
6

How Do Our Students Communicate with Other Healthcare Providers?

Buhler, Coplen, Davis, Nijjar
Journal of Research in Interprofessional Practice and Education

Vol. 6.2

2016

This was tested by analyzing for specific differences in the self-reported primary communication styles for each profession as well as mean scores for four communication style dimensions: Director, Presenter, Mediator, and Strategist. Additional analysis was performed for style dimension association with age, gender, and race. Achievement of the educational goal of the activity was measured by student completion of all the activities associated with the learning objectives.

Statistical analysis was performed using SPSS software (IBM SPSS v22.0). To determine if there were significant differences in PSCI component scores between programs, a series of one-way analysis of variance (ANOVA) analyses were conducted, followed by Tukey post-hoc analyses. To determine if there were significant differences in PSCI component scores between male and female students, a series of independent-sample $t$-test analyses were conducted. To determine if there was a significant relationship between participant age and PSCI dimension scores, a bivariate correlation procedure was conducted.

\section{Results}

\section{Participant demographics}

As this activity was a required component of the course, there was a 100 percent response rate, with a total of 1,198 students responding. In addition to the required components of PSCI and program affiliation (see Table 2a.), students were given the option to include additional demographic information. Of the total sample, 1,157 individuals provided information regarding gender identification (312 male, 845 female) and age (mean 27.0 years; range 19-59 years). In addition, 1,132 individuals provided information regarding race (see Table $2 \mathrm{~b}$.).

\section{Table 2a. Student demographics: Program affiliation}

\begin{tabular}{|l|c|c|}
\hline Program & Frequency & Percent \\
\hline Athletic Training & 20 & $1.7 \%$ \\
\hline Healthcare Administration & 38 & $3.2 \%$ \\
\hline Professional Psychology & 269 & $23 \%$ \\
\hline Speech-Language Pathology & 69 & $6 \%$ \\
\hline Physician Assisted Studies & 142 & $12 \%$ \\
\hline Audiology & 65 & $5 \%$ \\
\hline Dental Hygiene & 88 & $7 \%$ \\
\hline Occupational Therapy & 98 & $8 \%$ \\
\hline Physical Therapy & 134 & $11 \%$ \\
\hline Pharmacy & 275 & $23 \%$ \\
\hline Total & $\mathbf{1 , 1 9 8}$ & $\mathbf{1 0 0 \%}$ \\
\hline
\end{tabular}

Table 2b. Student demographics: Participant self-reported racial identity

\begin{tabular}{|l|c|c|}
\hline $\begin{array}{l}\text { Self-reported } \\
\text { racial identity }\end{array}$ & Frequency & Percent \\
\hline White & 831 & $73.4 \%$ \\
\hline Asian & 180 & $15.9 \%$ \\
\hline $\begin{array}{l}\text { Black or African- } \\
\text { American }\end{array}$ & 17 & $1.5 \%$ \\
\hline Asian-Indian & 24 & $2.1 \%$ \\
\hline $\begin{array}{l}\text { Native Hawaiian or } \\
\text { other Pacific Islander }\end{array}$ & 13 & $1.2 \%$ \\
\hline $\begin{array}{l}\text { American Indian or } \\
\text { Alaska Native }\end{array}$ & 10 & $0.9 \%$ \\
\hline Multi-racial & 57 & $5.0 \%$ \\
\hline Total & $\mathbf{1 , 1 3 2}$ & $\mathbf{1 0 0 \%}$ \\
\hline
\end{tabular}


7

How Do Our Students

Communicate with Other Healthcare Providers?

Buhler, Coplen, Davis, Nijjar

\section{PSCI dimensions and differences by program}

Primary affiliation, which describes the dimension ranked as highest by a student, indicated that a large majority of students (over 50 percent for all programs except the Masters of Healthcare Administration endorsed the Mediator dimension as their primary affiliation) (see Figure 1). In order to describe students' endorsements within all dimensions, all further analyses were done on mean scores by dimension reported here with standard deviation (SD). Mean scores indicate a strong preference for the Mediator dimension among the entire cohort: Director ( $8.50 \pm 3.77)$, Presenter ( $9.24 \pm 4.01)$, Mediator (12.56 \pm 4.23$)$, Strategist $(9.34 \pm 4.15)$.

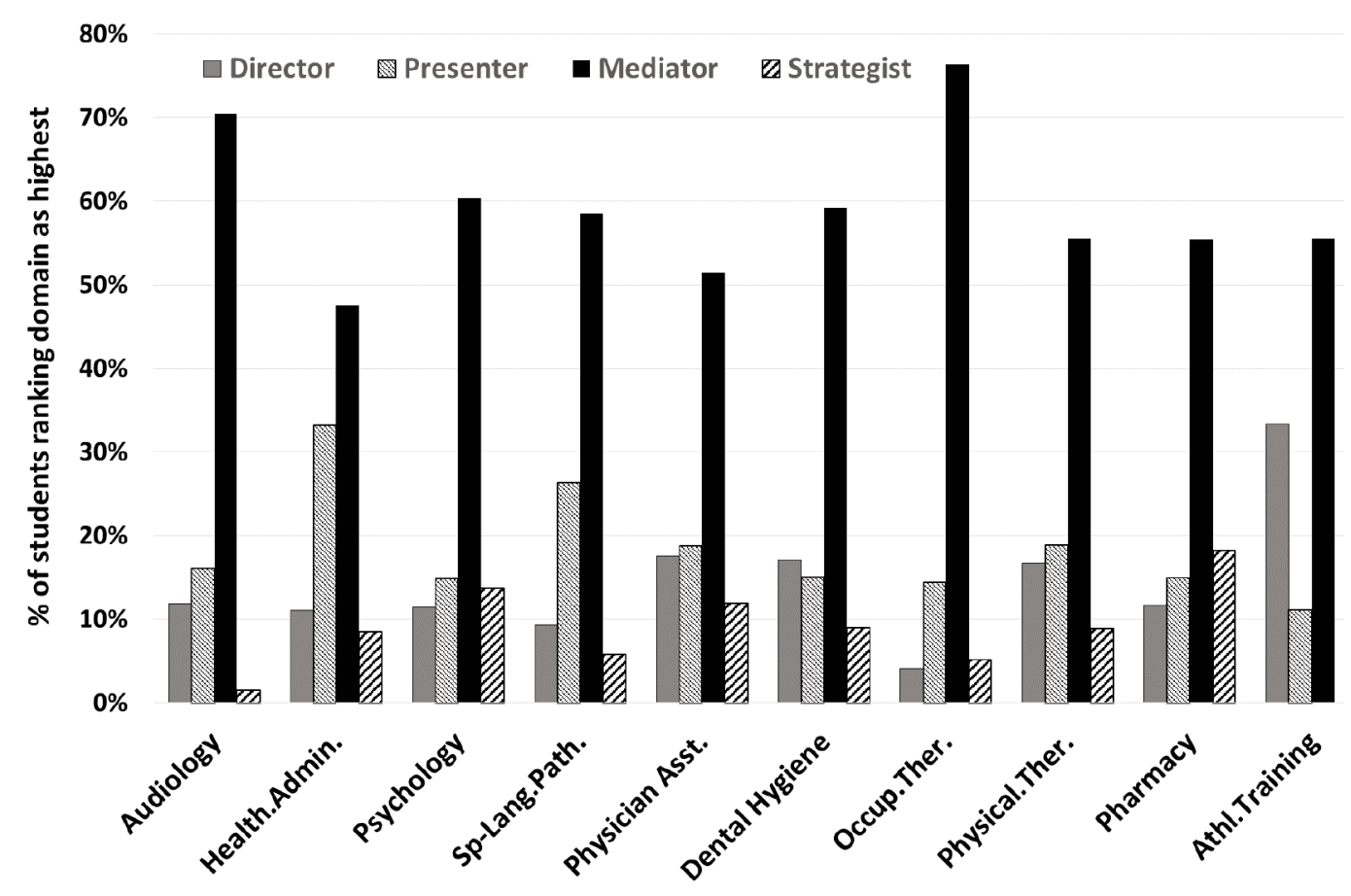

Figure 1. Primary dimension affiliation by program

To determine if there were significant differences in PSCI component scores between programs, a series of one-way ANOVA analyses were conducted. Means scores for each of the PSCI dimensions by program are presented (see Table 3). Significant differences between programs were found for the Director dimension $(F[9,1188]=2.53, p=.007)$, the Presenter dimension $(F[9,1188]=4.57, p<.001)$, and the Mediator dimension, $(F[9,1188]=5.79, p<.001)$. No significant differences in mean scores between programs were found for the Strategist dimension. Post-hoc Tukey analyses were conducted to determine which programs in particular differed and only items with significant differences $(p<0.05)$ are reported here. Post-hoc analyses revealed significant mean score differences in the Director, Mediator, and Presenter dimensions (Figures 2-4). No other significant mean differences were found. 


\section{JRIPE}

8

How Do Our Students

Communicate with Other Healthcare Providers?

Buhler, Coplen, Davis, Nijjar
Table 3. PSCI dimension mean scores and standard deviation (SD) by program

\begin{tabular}{|l|c|c|c|c|}
\hline Program & \multicolumn{1}{|c|}{ Director } & Presenter & \multicolumn{1}{c|}{ Mediator } & Strategist \\
\hline Athletic training & $9.2 \pm 4.0$ & $8.8 \pm 3.7$ & $11.9 \pm 4.6$ & $7.6 \pm 4.4$ \\
\hline Healthcare administration & $10.0 \pm 3.8$ & $8.3 \pm 4.3$ & $12.0 \pm 3.9$ & $10.4 \pm 3.5$ \\
\hline Professional psychology & $8.9 \pm 3.9$ & $9.4 \pm 4.2$ & $13.1 \pm 3.9$ & $9.4 \pm 4.2$ \\
\hline Speech-language pathology & $8.5 \pm 3.7$ & $10.3 \pm 3.9$ & $14.2 \pm 3.8$ & $9.4 \pm 3.5$ \\
\hline Physician assistant studies & $8.3 \pm 3.7$ & $9.1 \pm 4.0$ & $11.2 \pm 4.6$ & $8.5 \pm 4.2$ \\
\hline Audiology & $8.8 \pm 3.9$ & $10.1 \pm 3.9$ & $13.5 \pm 4.1$ & $9.6 \pm 4.2$ \\
\hline Dental health service & $7.7 \pm 3.5$ & $8.4 \pm 3.4$ & $12.1 \pm 3.7$ & $9.3 \pm 3.6$ \\
\hline Occupational therapy & $8.3 \pm 3.5$ & $10.4 \pm 3.7$ & $13.5 \pm 3.8$ & $9.2 \pm 4.1$ \\
\hline Physical therapy & $9.0 \pm 3.6$ & $10.0 \pm 4.0$ & $13.1 \pm 4.0$ & $9.3 \pm 4.1$ \\
\hline Pharmacy & $8.0 \pm 3.8$ & $8.4 \pm 3.9$ & $11.8 \pm 4.6$ & $9.7 \pm 4.4$ \\
\hline Total cohort (mean \pm SD) & $\mathbf{8 . 5} \pm \mathbf{3 . 8}$ & $\mathbf{9 . 2 \pm 4 . 0}$ & $\mathbf{1 2 . 6 \pm 4 . 2}$ & $\mathbf{9 . 3} \pm 4.2$ \\
\hline
\end{tabular}

Director

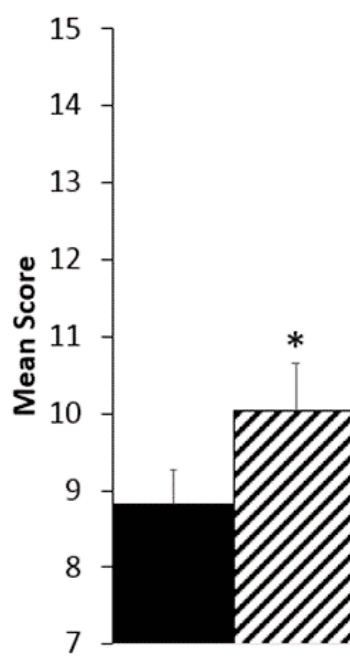

ournal of Research in Interprofessional Practice and Education

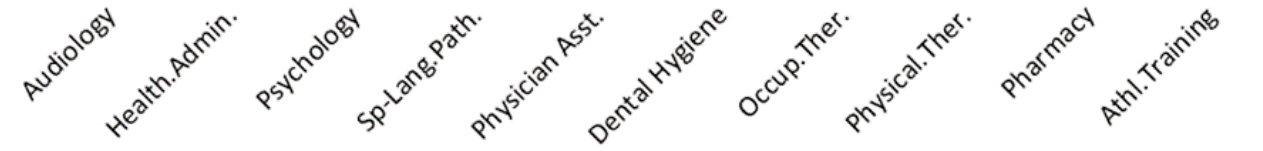

Figure 2. Program mean scores and SE for the Director dimension Note: Icons indicate significant differences of $p<0.05$ between programs. 
9

How Do Our

Students

Communicate with

Other Healthcare

Providers?

Buhler, Coplen,

Davis, Nijjar

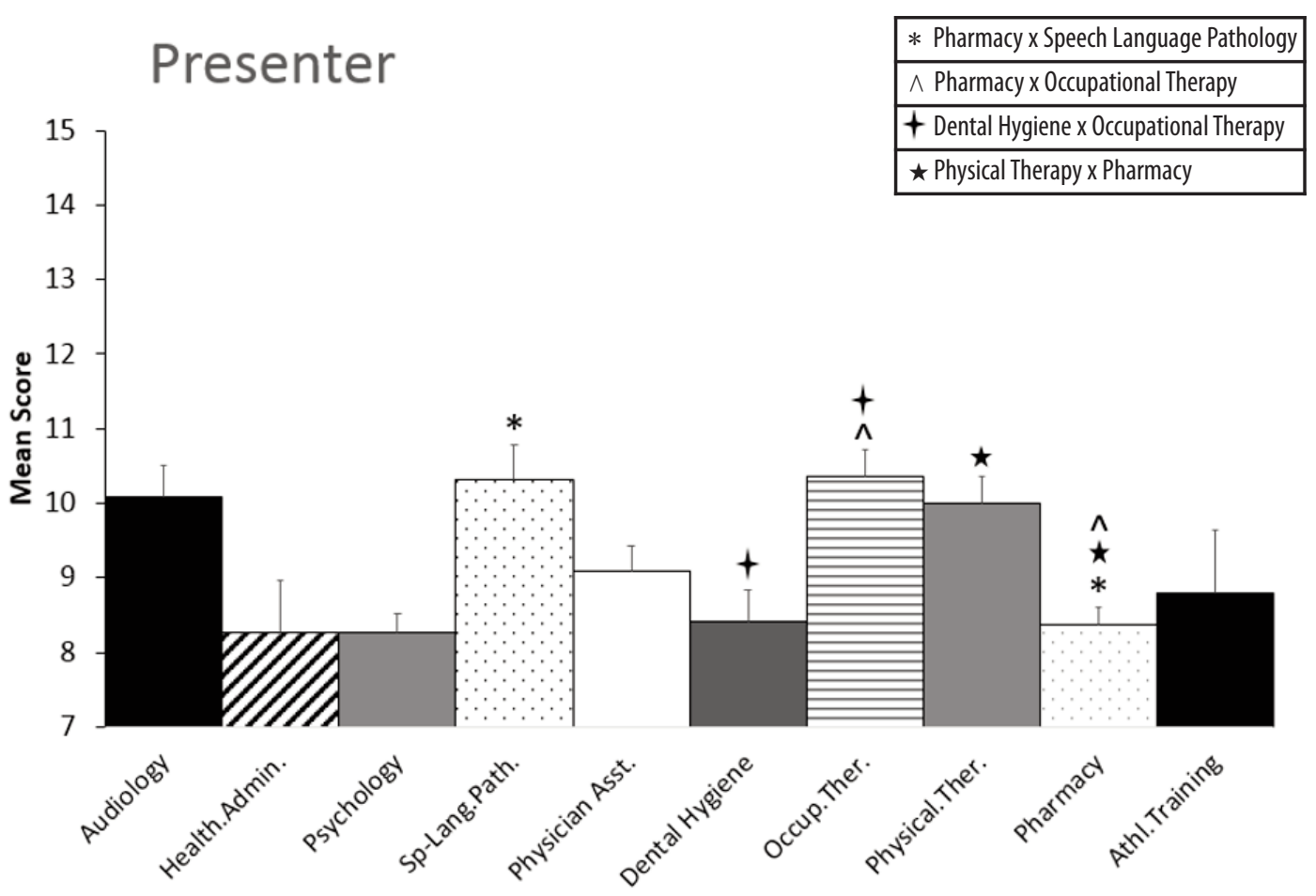

Figure 3. Program mean scores and SE for the Presenter dimension
Journal of Research in Interprofessional

Practice and

Education

Vol. 6.2

2016
Note: Icons indicate significant differences of $p<0.05$ between programs.

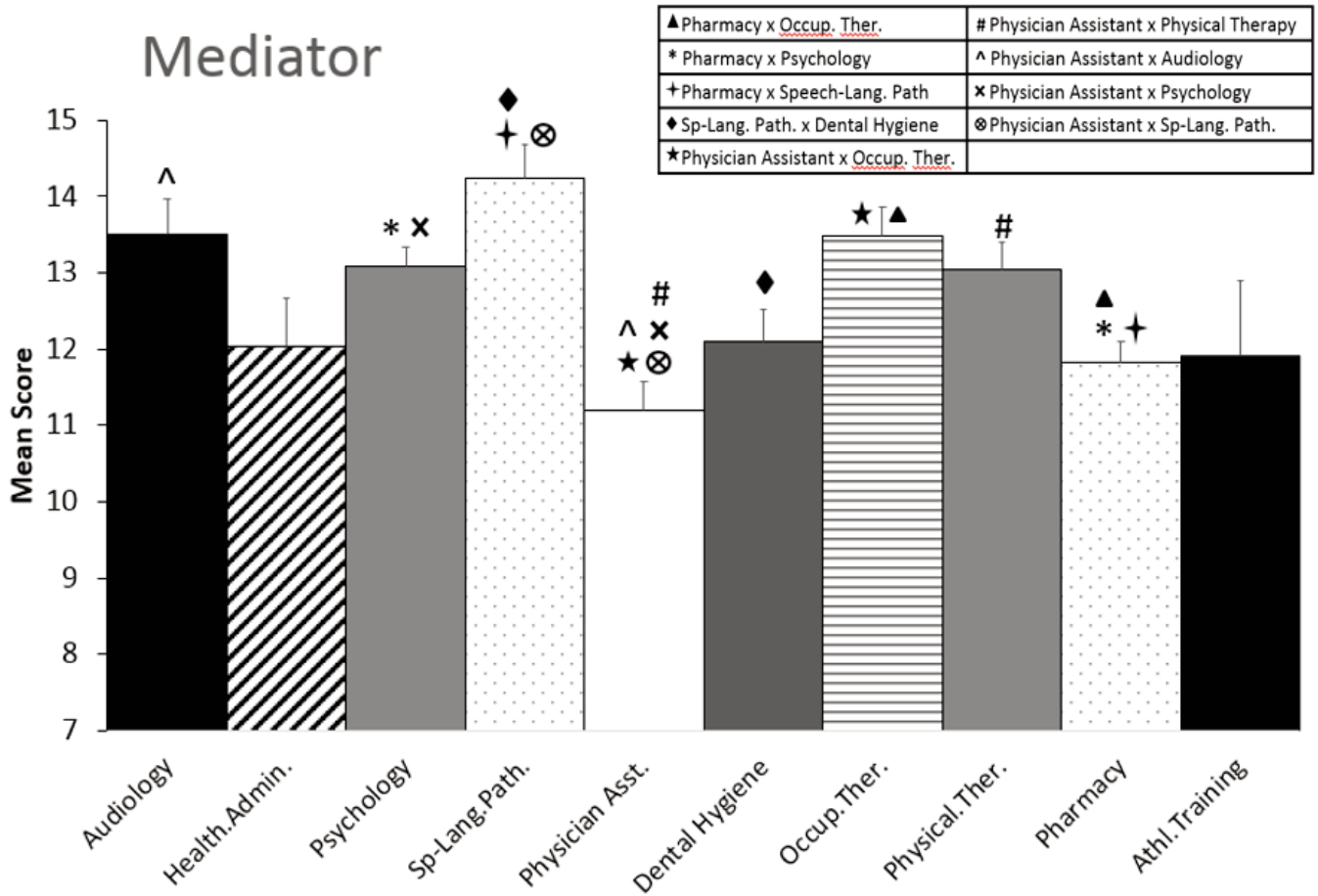

Figure 4. Program mean scores and SE for the Mediator dimension Note: Icons indicate significant differences of $p<0.05$ between programs. 
10

How Do Our Students Communicate with Other Healthcare Providers?

Buhler, Coplen, Davis, Nijjar
Journal of Research in Interprofessional Practice and Education

Vol. 6.2

2016

\section{PSCI differences by gender}

To determine if there were significant differences in PSCI component scores between male and female students (see Table 4), a series of independent-sample $t$-test analyses were conducted. A significant difference in mean scores within the Strategist dimension was found; $t(1155)=-2.19, p=.037$. No other significant differences between genders were found.

Age and PSCI dimension relationships To determine if there was a significant relationship between participant age and PSCI dimension scores, a bivariate correlation procedure was conducted. Across the entire

Table 4. PSCI dimension mean scores and SD by gender

\begin{tabular}{|l|c|c|}
\hline & Male $(n=312)$ & Female $(n=845)$ \\
\hline Director & $8.4 \pm 3.9$ & $8.6 \pm 3.7$ \\
\hline Presenter & $9.6 \pm 4.3$ & $9.1 \pm 3.9$ \\
\hline Mediator & $12.4 \pm 4.7$ & $12.7 \pm 4.0$ \\
\hline Strategist & ${ }^{*} 8.9 \pm 4.4$ & ${ }^{*} 9.5 \pm 4.0$ \\
\hline
\end{tabular}

* indicates significant difference in mean scores of $p<0.05$. sample, age was not found to be significantly related to scores on any of the PSCI dimensions.

\section{Race differences in PSCI dimensions}

Differences between self-reported races were found for the Director dimension only $(F[6,1140]=5.18, p<.001)$. Post-hoc analysis revealed this mean score difference in the Director dimension to be between: individuals identifying as White $(M=8.77)$ and those identifying as Asian $(M=7.40)$, and individuals identifying as Multiracial $(M=9.50)$ and those identifying as Asian $(M=7.40)$. No other significant differences between self-reported races were found.

\section{Discussion}

There is a wealth of literature citing communication problems in interprofessional healthcare teams, surprisingly, however, there are no reports investigating whether there are differences in communication styles between the professions. This is also the first report to describe a unit on individual communications styles as part of an interprofessional competence course. Determination of factors contributing to communication difficulties could help lead us to targets to intervene in or prevent suboptimal team communication patterns.

This communications activity had both a research question and an educational goal. The former was to determine whether differences in communication styles exist between students in different healthcare professions. The results of this study showed that student cohorts in all ten programs reported their highest scores in the PSCI Mediator dimension, which describes personable people, with expertise in their field, who instill trust in those who work with them. These are qualities consistent with the role of any healthcare provider, and it is not surprising that individuals with high scores in this dimension have chosen professions in these fields. However, it was of interest to determine whether students in different healthcare professions, who have varied job responsibilities and roles, would show any differences in communication styles. 
11

How Do Our Students Communicate with Other Healthcare Providers?

Buhler, Coplen, Davis, Nijjar

Journal of Research in Interprofessional Practice and Education

Vol. 6.2

2016
Data from this study suggest significant differences between professions on the Director, Presenter, and Mediator dimensions. Students in the Master of Healthcare Administration program had a higher percentage of Directors than any other program. Most of the students in this program are already full-time practitioners completing a second degree, and are seeking to move into a leadership role. This is consistent with the description of Directors as people who like to take charge, make good leaders, and are outspoken. One interesting finding was the low percentage of Physician Assistant students who self-identify as Directors. Previous studies show that MDs strongly self-identify as "leaders" and "decision makers" [17], and while trained in different professional cultures, Physician Assistants often function in a similar role as $\mathrm{MDs}$ on interprofessional teams [18]. It would be interesting to see whether Physician Assistants and MDs have different dynamics within interprofessional team functioning.

It is more difficult to speculate why significant differences were found in the Mediator dimension, as the majority of students identified as Mediators, and these skills appear to be integral to work in the health professions. It is also unclear what underlies the finding that students in Speech-Language Pathology, Occupational Therapy, and Physical Therapy had a higher percentage of Presenters in comparison with the other professions. Presenters are described as people who "like to have everything they do be fun," but it may be simplistic to speculate that this is connected to a draw toward professions that emphasize the demonstration of techniques and motivation of patients.

We found that female students reported higher mean scores in the Strategist dimension than male students. Only two studies have reported the impact of gender on communication style within health professions students. A study of dental students found males to have a higher score for friendliness while females had a higher teamwork score [19], and a study of medical students reported males to be more aggressive than females [20]. Strategists, as described by the PSCI, tend to be tacticians and diplomatic communicators. Our data is consistent with these findings and with studies in the social sciences that find that during group communication women tend to use strategies that minimize status distinctions and save face for the subordinate [21]. This has also been seen within physicians' and nurse practitioners' one-on-one communications with patients, in which interestingly, the strategies that minimized status differences were more effective in producing patient compliance [21].

We also found that students who identified as Asian had lower mean scores in the Director dimension than did other students. Directors, as described by the PSCI, tend to be assertive and outspoken. Consistent with our findings, multiple studies have suggested that Asian populations use more indirect communication styles [22]. However, there are two important caveats to consider when interpreting this data. First, variation between individuals in a culture is often greater than that between cultures [22], and second, it is important to note that the terminology "Asian" encompasses many different cultures with presumably heterogeneous communication traits. The majority of the students in the Health Professions Programs identifying as Asian descend from southeast Asian, particularly Vietnamese, ancestry. In any case, 
12

How Do Our Students Communicate with Other Healthcare Providers?

Buhler, Coplen, Davis, Nijjar

Journal of Research in Interprofessional Practice and Education

Vol. 6.2

2016 while being aware of group differences, it is important for practitioners to modulate communication style based on the individual communication preferences of their co-workers and patients rather than on their gender, race, culture, or profession.

As a result of the educational activity in this study, all learning objectives for the students were achieved: students used the survey to identify patterns within their own style of communication, they were exposed to differences in communication styles endorsed by other health professions, and, within their interprofessional small groups, they discussed the effect their style has on communication with others. Finally, they considered the impact of heterogeneous communication styles within a clinical team. During this activity, the authors were able to observe student groups as they discussed their communication styles, and were pleased to find that students were engaged in the activity. Open-ended written evaluations collected at the end of that section were positive and indicated that students found the exercise to be thought provoking, and they were surprised at the prevalence of the Mediator dimension in all fields. These comments suggest that students value activities of selfreflection on personal communications styles and the discussion of the effects on interprofessional interactions.

Part of the responsibility of the educational bodies for healthcare professionals is to help students to develop "soft-skills" in addition to their profession-specific knowledge. It is unclear at this time whether it is more effective to teach awareness of communication skills early in the educational process than to attempt to intervene after practitioners have established set behavioural patterns. A few studies of IPE courses for post-graduate practitioners addressing interpersonal communications skills have produced self-reported improvements in "communications with colleagues" up to three to six months after completion $[23,24]$. Yet there is some literature that states the long-term benefit of team-based training workshops for licensed providers is weak [25,26]. It is to be hoped that early learning would establish good teamwork skills and decrease professional "siloing." It is likely that the hierarchical roles traditionally held by different professions have encouraged certain teamwork and conflict styles [27]. If this is the case, the expanding roles of the various professions may also encourage more diverse communication styles. It is anticipated that activities done during this course will provide students insight into their own communication styles, and in turn, will eventually translate into improved efficacy of team communications, both in class and in the field. It will be necessary, however, for future studies to confirm whether the differences between professions in this study in fact reflect real-world differences in communication dynamics.

While the PSCI provides useful information on general communication styles, with the increase in studies in interprofessional communications, it may be beneficial for future researchers to develop an inventory specifically designed for health professionals. A strength of the current study is that all ten professions utilized the same inventory. Most communications and conflict strategy studies in healthcare have studied only one or two professions, and have used varying inventories, making it difficult to compare findings between multiple professions. For these reasons, development and use of a standardized communication inventory for healthcare professionals would be beneficial to this field. 
How Do Our Students Communicate with Other Healthcare Providers?

Buhler, Coplen, Davis, Nijjar
Journal of Research in Interprofessional Practice and Education

Vol. 6.2

2016

\section{Conclusions and recommendations for future research}

In this article, the authors identified differences in communications styles of students within ten different Health Professions Programs. The differences found in this study may impact communication efficacy within interprofessional healthcare teams. This study is the first to look at communication styles within a large interprofessional student cohort. While several health professions were studied herein, health professionals in other fields, such as nursing and medicine, could also benefit from understanding how their personal communication style may vary from their interprofessional care team members, and how this may impact teamwork in the clinical setting. Determining the factors that contribute to communication insufficiencies could help direct us to targets for education designed to intervene in or prevent negative team communication patterns. These findings suggest the need for additional investigations of the impact of pre- or post-graduation interpersonal communications training on interprofessional practice clinical outcomes.

\section{Acknowledgements}

Our thanks to Amy L. Johnston for expert editing assistance and to CoachWorks International (Dallas, Texas, US) for permission to use the PSCI inventory.

\section{References}

1. Interprofessional Education Collaborative. (2011). Core competencies for interprofessional collaborative practice: Report of an expert panel. Washington, DC: Interprofessional Education Collaborative.

2. Van Leijen-Zeelenberg, J.E., Van Raak, A.J., Duimel-Peeters, I.G.P., Kroese M., Brink P, Vrijhoef, H. (2015). Interprofessional communication failures in acute care chains: How can we identify the causes? Journal of Interprofessional Care, 18(20), 1-11.

3. de Vries, R.E., Bakker-Pieper, A., Alting Siberg, R., van Gameren, K., \& Vlug, M. (2009). The content and dimensionality of communication styles. Communication Research, 36(2), 178-206.

4. Bach, S., \& Grant, A. (2009). Communication and interpersonal skills for nurses. Exeter, England: Learning Matters.

5. van Ess Coeling, H., \& Cukr, P. (2000). Communication styles that promote perceptions of collaboration, quality, and nurse satisfaction. Journal of Nursing Care Quality, 1(14), 63-74.

6. Zwarenstein, M., Rice, K., Gotlib-Conn, L., Kenaszchuk, C., \& Reeves, S. (2013). Disengaged: A qualitative study of communication and collaboration between physicians and other professions on general internal medicine wards. BMC Health Services Research, 13(1), 494.

7. Hewitt, G., Sims, S., \& Harris, R. (2015). Evidence of communication, influence and behavioral norms in interprofessional teams: A realist synthesis. Journal of Interprofessional Care, 29(2), $100-105$.

8. Aritzeta, A., Ayestaran, S., \& Swailes, S. (2005). Team role preference and conflict management styles. International Journal of Conflict Management, 16(2), 157-182.

9. de Vries, R., van den Hooff, B., \& de Ridder, J. (2006). Explaining knowledge sharing. Communication Research, 33(2), 115-135.

10. Somech, A. (2006). The effects of leadership style and team process on performance and innovation in functionally heterogeneous teams. Journal of Management, 32(1), 132-157.

11. Cho, H., Gay, G., Davidson, B., \& Ingraffea, A. (2007). Social networks, communication styles, and learning performance in a CSCL community. Computers \& Education, 49(2), 309-329.

12. Brock, D., Abu-Rish, E., Chiu, C.R., Hammer, D., Wilson, S., Vorvick, L., Blondon, K., Schaad, D., Liner, D., \& Zierler, B. (2013). Interprofessional education in team communication: Working together to improve patient safety. BMJ Quality and Safety, 22(5), 414-423.

13. Salvatori, P., Mahoney, P., \& Delottinville, C. (2006). An interprofessional communication skills lab: A pilot project. Education for Health, 19(3), 380-384.

14. Coachworks International. (1999). Personal Coaching Style Inventory. URL: http://coachworks .com/magento/ [June 9, 2015]. 
14

How Do Our Students Communicate with Other Healthcare Providers?

Buhler, Coplen, Davis, Nijjar
15. Moodle. (2016). Moodle Platform. URL: http://www.pacificu.edu/libraries/educational-technology /moodle [March 20, 2016].

16. NIH grants guidelines. (2015). NIH policy on reporting race and ethnicity data: Subjects in clinical research. URL: http:/grants.nih.gov/grants/guide/notice-files/NOT-OD-01-053.html [March 20, 2016].

17. Baker, L., Egan-Lee, E., Martimianakis, M.A.T., \& Reeves, S. (2011). Relationships of power: Implications for interprofessional education. Journal of Interprofessional Care, 25(2), 98-104.

18. American Academy of Family Physicians and American Academy of Physician Assistants. (2011). Family physicians and physician assistants: Team-based family medicine, a joint policy statement. URL: http://www.aafp.org/dam/AAFP/documents/practice_management/admin_staffing/AAFP -AAPAJtPaper.pdf [March 20, 2016].

19. Silberman, S., Freeman, I., \& Lester, G. (1992) A longitudinal study of dental students' personality type preferences. Journal of Dental Education, 56(6), 384-388.

20. Lee, K., Vaishnavi, S., Lau, S., Andriole, D., \& Jeffe, D. (2009). Cultural competency in medical education: Demographic differences associated with medical student communication styles and clinical clerkship feedback. Journal of the Nationall Medical Association, 101(2), 116-126.

21. Kendal, S., \& Tannen, D. (1997). Gender and discourse. London, UK: Sage Publications.

22. Park, H.S., Levine, T., Weber, R., Lee, H.E., Terra, L., Botero, I., Bessarabova, E., Guan, X., Shearman, S., \& Wilson, M.S. (2012). Individual and cultural variations in direct communication style. International Journal of Intercultural Relations, 36(2), 179-187.

23. Sargeant, J., MacLeod, T., \& Murray, A. (2011). An interprofessional approach to teaching communication skills. Journal of Continuing Education in the Health Professions, 31(4), 265-267.

24. Norgaard, B., Ammentorp, J., Ohm, K., \& Kofoed, P.E. (2012). Communication skills training increases self-efficacy of health care professionals. Journal of Continuing Education in the Health Professions, 32(2), 90-97.

25. Lee, P., Allen, K., \& Daly, M. (2015). A “communication and patient safety” training programme for all healthcare staff: Can it make a difference? BMJ Quality \& Safety 21(1), 84-89.

26. McCulloch, P., Rathbone, J., \& Catchpole, K. (2011). Interventions to improve teamwork and communications among healthcare staff. British Journal of Surgery, 98(4), 469-479.

27. Wright, K. (2011). A communication competence approach to healthcare worker conflict, job stress, job burnout, and job satisfaction. Journal for Healthcare Quality, 33(2), 7-14. 